\title{
LA EMERGENCIA DEL COACHING COMO PROFESIÓN EN UN CONTEXTO LABORAL DE OPORTUNIDAD Y VULNERABILIDAD ${ }^{1}$
}

Idoia Gorroño Arregui Socióloga y PNL Coach info@arreguicoach.com

Recibido: 28-02-09

Aceptado: 18-03-09

\section{Resumen}

En este texto se reflexiona acerca de los cambios y paradojas que están teniendo lugar en el mundo del trabajo, las organizaciones y los profesionales. Es este contexto, en el que el trabajo incorpora las emociones como competencia, el que puede posibilitar la emergencia del coaching como profesión en España. En primer lugar, se abordará la paradoja de la era flexible como oportunidad y vulnerabilidad para los profesionales. En segundo lugar, las competencias emocionales como ventaja competitiva y éstas, a su vez, como riesgo. En tercer lugar, el límite cada vez más difuso entre el ámbito laboral y doméstico para, finalmente, subrayar algunas contradicciones en torno a las relaciones de género en las organizaciones. En este transito entre la vulnerabilidad y la oportunidad en el contexto organizativo emerge el ámbito de intervención del coaching, que acompaña a las personas y profesionales en el desarrollo de sus habilidades y competencias con el propósito de aumentar su rendimiento y calidad de vida.

\footnotetext{
${ }^{1}$ Agradezco profundamente a Maite Martín Palomo, mentora en el difícil proceso de escribir, por el acompañamiento, la confianza y los aportes recibidos. También a los diversos profesionales de los que me he nutrido en conferencias y espacios de reflexión organizados por ICF (Federación Internacional de Coaching) y AECOP (Asociación Española de Coaching y Consultoría de Procesos).
} 
Palabras clave: Era flexible, organizaciones, competencias emocionales, paradojas, vulnerabilidad profesional, coaching.

\begin{abstract}
This paper reflects on the changes and paradoxes that are taking place in the labor world, in the organizations and in the professionals. It is this context, in which the labor incorporates emotions and communication skills as competences that may enable the emergence of coaching as a profession in Spain. Firstly, we will address the paradox of the flexible era as an opportunity and vulnerability to the professionals. Secondly, emotional competencies as competitive advantage and, in turn, as a risk. Thirdly, the increasingly diffuse boundary between the workplace and home, to finally highlight some contradictions about gender relations in organizations.
\end{abstract}

Key words: Flexible Era, organizations, emotional competencies, paradoxes, professional vulnerability, coaching.

\title{
1. Contexto del ámbito del trabajo: la era flexible
}

Los estudios sociológicos recientes, desde diversas perspectivas, definen la sociedad de comienzos del siglo XXI como sociedad post-industrial (Bell, 1970), sociedad del riesgo (Beck, 2006), sociedad de la información (Castells, 2001) o la sociedad del hiperconsumo (Lipovetsky, 2007). Estas teorías describen fenómenos como la globalización, el desarrollo de las nuevas tecnologías de la información y la comunicación, los nuevos sistemas de terciarización y producción de la economía y la sociedad del conocimiento. Siguiendo a Boltansky y Chiapello, un fenómeno que atraviesa el contexto actual es la denominada era flexible que, desarrollándose en contextos de incertidumbre, puede ser analizada, tanto por sus componentes externos (la internacionalización de la economía y la deslocalización), como por los internos, es 
decir, por las profundas transformaciones de la organización del trabajo, sustentadas a su vez en cambios ideológicos (Boltansky y Chiapello, 2002: 33).

Según estos autores, la flexibilidad sería uno de los ejes principales de la nueva estrategia empresarial caracterizada por valores como la innovación, la creatividad, el cambio permanente, el autocontrol, la polivalencia y el desarrollo de la autonomía, que es interiorizado por las organizaciones y también, como veremos más adelante, por los profesionales (Boltansky y Chiapello, 2002).

Una de las ideas-fuerza de estos autores es la noción de proyecto ${ }^{2}$ que sustituye la idea de carrera profesional ${ }^{3}$ por la sucesión de un proyecto profesional a otro, de tal forma que el éxito obtenido en uno de ellos les permita acceder a otros en los cuales se pueda generar valor nuevamente. El acceso a estos proyectos se complementa a través del aumento del nivel de empleabilidad, es decir, de la capacidad de pasar de uno a otro, lo que otorga seguridad en esta concepción del universo del trabajo flexible, móvil y en cambio permanente (Boltansky y Chiapello, 2002: 147).

Las organizaciones, a su vez, se adaptan a estas transformaciones del trabajo y de la sociedad contemporánea. Se hacen más flexibles, abiertas, horizontales y desarrollan modalidades de trabajo en red (Giddens, 2001). En esta inercia también pueden solicitar a los profesionales todas sus capacidades para desarrollarse en este nuevo marco. Capacidades como la escucha, el liderazgo, la creatividad, la empatía y el trabajo en equipo se constituyen en competencias cuando se convierten en valor y en ventaja competitiva para ampliar el nivel de empleabilidad y transitar de un proyecto a

\footnotetext{
${ }^{2}$ La noción de proyecto se instala a partir de la década de los 90 y contempla la realización profesional mediante el trabajo en diversos proyectos que pueden ser dentro de la misma organización u otras. En realidad implica un desarrollo de la autonomía del profesional, salvando las barreras también jerárquicas, geográficas gracias al desarrollo de las tecnologías de la información y a este modelo de gestión empresarial. Esta noción de proyecto no sería posible sin el desarrollo de la flexibilidad que también implica un aumento de la precariedad laboral en cuando a condiciones de trabajo.(Boltansky y Chiapello, 2002).

${ }^{3}$ Según estos autores, la noción de carrera profesional es característica del segundo espíritu del capitalismo, propio de la sociedad industrial, en el que los trabajadores dedican su tiempo de trabajo generalmente a la misma empresa y en el mismo sector.
} 
otro. Estas competencias emocionales y conversacionales ${ }^{4}$ (Echeverría, 2006) permiten liderar las organizaciones del siglo XXI y hacen que los profesionales se sientan parte de un proyecto organizativo, se impliquen en el desarrollo del mismo y puedan convertir sus cualidades en fuerza de trabajo que genere a su vez valor en la organización (Boltansky y Chiapello, 2002).

Ahora bien, frente a la tradicional organización del trabajo, donde la vida privada y la vida pública estaba separada y simbolizada en la casa y la fábrica, caracterizada a su vez por la división sexual del trabajo, en la nueva organización del trabajo, también asentada en la denominada como modernidad líquida, las fronteras se desdibujan, siendo estas cada vez más flexibles, móviles y ambiguas (Bauman, 2005). Todo ello, además configura las relaciones de género en las organizaciones, donde las mujeres debido a, su nivel cada vez más elevado de cualificación, su paulatina incorporación a profesiones consideradas masculinas, tienen un nivel de participación y presencia también en los distintos niveles de las organizaciones, no sin contradicciones como se describirá más adelante (Sarrió, 2008: 184).

Así pues, es en este contexto donde tiene cabida la noción de proyecto, la empleabilidad y la era flexible. Tal y como lo describe Bauman, la modernidad líquida sustentada en la sociedad de consumo degrada los ideales del largo plazo (Bauman, 2005: 65). El tiempo líquido es el tiempo del aquí y el ahora, de la inmediatez, el tiempo del cambio permanente.

Ahora bien, además de en la cultura y el clima de las organizaciones, estos valores van calando en la psicología de los profesionales, tanto mujeres como hombres, que los vuelven suyos como antídotos a la gestión de la incertidumbre y los riesgos (Beck, 1998). En este tiempo líquido, la subjetividad de los profesionales se pone a trabajar (Hochschild, 2008) e involucra el universo emocional y afectivo, pudiéndose

\footnotetext{
${ }^{4}$ El concepto de competencia conversacional es desarrollado por Rafael Echeverría. Este autor considera las organizaciones como redes conversacionales que crecen a través de las conversaciones en un contexto de globalización y flexibilidad. Las conversaciones, en contextos de relaciones y a través del poder generativo del lenguaje, son las que producen la acción. Este concepto se basa en los estudios de Fernando Flores, Humberto Maturana y Francisco Varela.
} 
generar importantes paradojas que transitan entre la oportunidad y la vulnerabilidad de los mismos. En la era flexible se les pide que sean ágiles, que se adapten al cambio, que asuman riesgos, por lo que se agotan en sí las trayectorias lineales y se desarrollan fragmentos de trabajo (Sennet, 2000: 9) que tienen implicaciones emocionales como la ansiedad, miedo y soledad, antesalas de la vulnerabilidad (Sennet, 2000).

¿Pero cuáles son estas paradojas? ¿Cuáles las oportunidades y vulnerabilidades de este escenario flexible? ¿Cuáles son las contradicciones de género en las organizaciones? ¿Por qué entonces es necesario hablar de la emergencia del coaching?

\section{Algunas paradojas en el contexto organizativo}

\subsection{Primera paradoja: entre la oportunidad y la vulnerabilidad ${ }^{5}$}

Las oportunidades que son generadas en la era flexible implican una necesidad de reinvención constante del individuo en contextos de gran incertidumbre e innovación, el desarrollo del potencial en base a competencias, no solo técnicas, sino también emocionales y relaciones (Echeverría, 2006), el aprendizaje continuo y el trabajo en red. Todos estos procesos van de la mano del desarrollo de trayectorias profesionales diversas ${ }^{6}$ y fragmentadas frente a las trayectorias lineales tradicionales.

\footnotetext{
${ }^{5}$ Se define la vulnerabilidad como la cualidad del vulnerable, "aquel que puede ser herido física o moralmente" (RAE). La era flexible implica la vulnerabilidad en cuanto a las condiciones de trabajo, es decir, en un aumento de la precariedad (Boltansky y Chiapello, 2002). En este artículo, la autora la presenta en términos de vulnerabilidad intangible, aquella que tiene que ver con los sentimientos y las emociones.

${ }^{6}$ A pesar de que la reinvención puede ser una oportunidad, en ocasiones el motor que mueve esta diversidad profesional tiene que ver con fenómenos como la insatisfacción laboral y la precariedad laboral. A la vez las personas más preparadas para el cambio de profesión son aquellas que han ocupado puestos de responsabilidad y que además no tienen un nivel de especialización elevado que les permita adquirir la flexibilidad necesaria para realizar el transito a otra profesión con la dosis necesaria de planificación y entusiasmo (Méndez, 2009).
} 
Se plantea así la posible primera paradoja de la era flexible. La oportunidad de la reinvención constante, con todo lo que ello implica de libertad y creatividad, unida también a la vulnerabilidad de sentirse fragmentado, solo y aislado.

En la obra de Sennet “La corrosión del carácter”, esta paradoja está representada en un personaje, Rico ${ }^{7}$. Él simboliza el éxito del profesional de la era flexible: consultor autónomo, independiente, con una retribución económica importante e icono de la movilidad social ascendente en comparación con su familia primaria. Sin embargo, la otra cara de la moneda son los costes personales de su movilidad y flexibilidad profesional que intentan ser compensadas a través de las comunicaciones virtuales no del todo satisfactorias (Sennet, 2000: 19).

En definitiva, frente a oportunidades como la movilidad geográfica y social, el aprendizaje continuo, el desarrollo de la autonomía y creatividad, se convive con vulnerabilidades asociadas a la desarticulación social y familiar. Fundamentalmente, debido a la continua rotación y la despersonalización de las relaciones, que pueden generar pérdida de vínculos estables (Kaufmann, 2008: 28).

El desafío de Rico, y a la vez su vulnerabilidad, es tratar de conservar, en esa situación, su vida interior y emocional y la gestión del tiempo con su familia, sin que además ésta tenga que adaptarse al tiempo del trabajo (Sennet, 2000: 19).

\subsection{Segunda paradoja: las emociones como competencia versus riesgos}

Se ha expuesto que, en la nueva organización del trabajo, las capacidades de relación, las actitudes y valores forman parte de las competencias que los profesionales requieren para afrontar la vida por proyectos. Así pues, las emociones componen algunas de las competencias profesionales actuales. No es casual, por lo tanto, el

\footnotetext{
${ }^{7}$ En la obra citada, Sennet describe los cambios del capitalismo flexible en el ámbito del trabajo a través de la historia de vida de personas con el objetivo de explicar las consecuencias personales de la nueva organización del trabajo.
} 
aumento de publicaciones sobre management ${ }^{8}$, además de formaciones especializadas en las distintas escuelas de negocios y universidades. Unido a ello, el conocido concepto de inteligencia emocional, basado en los libros de Daniel Goleman ${ }^{9}$ y su impacto en la capacidad de trabajo (Kaufmann, 2003: 34).

Ahora bien, la gestión de las emociones también muestra indicadores de riesgo que bien pueden ser ilustrados mediante los siguientes estudios. La "Encuesta nacional de condiciones de trabajo" (Ministerio de Trabajo y Asuntos Sociales, 2007) revela que para un $21,5 \%$ de los profesionales las exigencias emocionales son percibidas como carga de trabajo (frecuencia de 'siempre o casi siempre'), frente a un $22,1 \%$ en términos de exigencias intelectuales en la misma frecuencia temporal. Este concepto de exigencias emocionales entendido como carga de trabajo ilustra la incorporación de las emociones en el trabajo más cercanas a la vulnerabilidad que a la competencia.

Por otro lado, en la "Encuesta de la calidad del trabajo" (Ministerio de Trabajo y Asuntos Sociales, 2006), casi el 75\% de los trabajadores consideran que tienen estrés, es decir, tres de cada cuatro personas que desarrollan un trabajo remunerado se encuentran en esta tesitura.

También, en el VI Informe Cisneros se recoge que el 9,2\% de los profesionales han padecido moobing ${ }^{10}$, con un $23 \%$ habiéndolo padecido en su trayectoria anterior y aumentando en un $70 \%$ el mobbing del jefe hacia el empleado.

\footnotetext{
${ }^{8}$ El management o gestión empresarial agrupa fundamentalmente textos sobre la organización empresarial. No se centran únicamente sobre aspectos técnicos sino que tratan también principios más generalistas, cercanos a principios de acción o filosofía de trabajo (Fernández, 2007).

${ }^{9}$ En su best seller "La inteligencia emocional" y libros posteriores, Daniel Goleman describe cómo las competencias más relevantes en el ámbito laboral son las asociadas a la inteligencia emocional, tales como la autoconciencia, la autoestima, el autocontrol, la empatía, la dedicación, la integridad y la habilidad para comunicar (Goleman, 1998).

${ }^{10} \mathrm{El}$ acoso moral o mobbing ha sido ampliamente estudiado por Iñaki Piñuel, autor del VI Informe Cisneros. En el mismo, se entiende por mobbing: "El continuo o deliberado maltrato verbal o modal que recibe un trabajador, con objeto de apocarlo o destruirlo psicológicamente y que consiste en comportamiento de hostigamiento o violencia psicológica frecuentes, recurrentes y sistemáticos". Visitado el 20 de Febrero de 2009. www.acosomoral.org.
} 
Finalmente, y en relación específica a las mujeres, en el reciente estudio cualitativo sobre las mujeres directivas, se observa fenómenos como el "síndrome de la mujer cansada" $" 11$ y emociones como el miedo y la culpa que pueden desencadenar en una baja autoestima y que son provocadas por el contexto organizativo en el que se mueven los profesionales y también por las desigualdades de género que aún persisten en las mismas (Kaufmann, 2007: 135).

Así pues, esta segunda paradoja plantea la versión problemática de las emociones como riesgos y, a su vez, como competencia de los profesionales, como valor y ventaja competitiva en esta sociedad compleja y del conocimiento, fundamentalmente para los puestos directivos y de responsabilidad.

Si bien podría profundizarse en el análisis de la dualización del mercado de trabajo y los segmentos profesionales, lo interesante es que las emociones pueden tener distintas connotaciones para los profesionales en el contexto organizativo, en una visión de la competencia como oportunidad y como riesgo de vulnerabilidad.

Es éste contexto de cambio y de reinvención del propio concepto de trabajo ${ }^{12} \mathrm{el}$ que posibilita la aparición del coaching como profesión caracterizada por acompañar y entrenar las competencias emocionales, de liderazgo y de comunicación en los profesionales. Sin embargo, dicha profesión también tendrá en cuenta dos aspectos fundamentales, por un lado, los límites difusos entre el ámbito doméstico y el ámbito del trabajo que a su vez también guardan relación con las contradicciones de género en el contexto organizativo y que no se pueden obviar. Estos aspectos configuran el resto de paradojas de la era flexible.

\footnotetext{
${ }^{11}$ En ocasiones, las propias exigencias internas derivadas de la complejidad de la conciliación de la vida personal y profesional, hace que se llegue a niveles de agotamiento más allá de lo soportable, que pueden terminar en crisis de ansiedad. (Kaufmann, 2007: 42).

${ }_{12} \mathrm{Al}$ hilo de los cambios en el concepto de trabajo en la era flexible, ya se ha planteado la propuesta teórica de domesticar el trabajo, es decir, revisar el concepto de trabajo para integrar en él los aspectos relacionados con el cuidado, la subjetividad y las emociones (Martín-Palomo, 2008).
} 


\subsection{Tercera paradoja: los límites líquidos entre la casa y el trabajo}

Los límites también difusos entre el ámbito profesional y el ámbito doméstico hace que se incorpore el trabajo a casa y se pueda hablar de la mercantilización de la vida íntima ${ }^{13}$ (Hochschild, 2008), lo que genera a su vez nuevas fronteras temporales que tienen impacto en la gestión del tiempo de las personas.

El desarrollo de las tecnologías de la información y la comunicación en la organización del trabajo se extienden los horarios laborales a través de la práctica del teletrabajo, el desarrollo del net-working ${ }^{14}$ virtual y físico y, con ello, la disponibilidad permanente de los profesionales a través del teléfono móvil, Internet, el portátil y el correo electrónico. Se instala así en el ámbito doméstico la denominada frontera temporal 24/7, esto es, la disponibilidad permanente, las 24 horas del día los 7 días de la semana. Esto genera que los profesionales en el ámbito laboral, y las mismas personas en el ámbito doméstico, sientan que disponen de menos tiempo, situación que se puede conectar con enfermedades como el estrés y la falta de sueño (Kaufmann, 2008: 27).

Este límite difuso entre el ámbito laboral y el ámbito doméstico implica debates más profundos, como la conciliación laboral ${ }^{15}$, la organización del trabajo y las

\footnotetext{
${ }^{13}$ Hochschild desarrolla el concepto de mercantilización de la vida intima por los servicios que son ofrecidos por el mercado a las tareas tradicionalmente cubiertas por la familia, tales como los servicios de asistentes personales, planificadores de fiestas. La autora toma como referencia la sociedad norteamericana. Además, se produce un traslado e inclusión de expresiones y lenguajes del mundo laboral al doméstico, por ejemplo, "invertir en la familia" (Hochschild, 2008: 66).

${ }^{14}$ El net-working es una práctica de trabajo propia de la sociedad del conocimiento, donde distintos profesionales intercambian conocimientos, informaciones e incluso pueden desarrollar ideas de negocio. Es un espacio libre, flexible y en red que se puede práctica presencialmente o través de las tecnologías de la información.

${ }^{15}$ La conciliación laboral relaciona el trabajo, la sociedad y la familia como un puzzle complejo de armonizar. Pone en cuestión la dedicación plena y exclusiva al trabajo, desarrollando áreas como el tiempo dedicado al desarrollo personal, familiar y social, desde una visión de responsabilidad, oportunidad y disfrute. La conciliación laboral en el ámbito doméstico, y en relación con las tareas reproductivas, tiene una implicación directa con las relaciones de género. Estas reflexiones en torno a la conciliación han sido tomadas de la conferencia en la Asociación Española de Consultoría de Procesos (AECOP) sobre "Conciliación como activo estratégico de las organizaciones", en particular de las reflexiones de Alicia Kaufmann, Eva Levy y Ana Moreno.
} 
relaciones de género en el contexto profesional al que dedicaremos el apartado siguiente.

\subsection{Cuarta paradoja: algunas contradicciones de género a tener en cuenta}

Las organizaciones actuales requieren de los profesionales unas competencias emocionales ${ }^{16}$ que conforman un estilo de liderazgo más en consonancia con valores asociados tradicionalmente a las mujeres (Cuadrado, 2006: 51) ${ }^{17}$. Por ello, el conjunto de competencias que son requeridas en la era flexible configura un tipo de liderazgo de características comunales y expresivas, estereotípicamente femeninas, frente a un estilo de liderazgo más agéntico o instrumental, estereotípicamente masculino (Cuadrado, 2006: 35).

En el estudio cualitativo mencionado anteriormente se contempla que las mujeres que, o bien han ejercido el cuidado, o bien han sido madres, han desarrollado capacidades y habilidades emocionales que benefician la relación de las personas dentro de la organización y facilitan la gestión para trabajar en equipo (Kaufmann, 2007: 43). Sin embargo, no es menos cierto que las mujeres en el ámbito profesional asumen numerosas barreras basadas en los estereotipos y expectativas de género ${ }^{18}$.

También se ha analizado que el desarrollo de la identidad a nivel de género es construido socialmente de distinta manera para hombres y para mujeres, por lo que

\footnotetext{
${ }^{16}$ Las competencias emocionales contemplan un conjunto de habilidades, como el distinguir espacios emocionales y el saber comprender las distintas emociones de las personas y de los equipos distinguiéndolas de las propias (Echeverría, 2006).

${ }^{17}$ Esta demanda de liderazgo está relacionada también con el cambio en los modelos teóricos de de las personas en las organizaciones. En la década de los 80 se habla de "Recursos Humanos" mientras que en los primeros años del siglo XXI se habla de "Dirección y desarrollo de personas" (Gorroño, 2008).

${ }^{18}$ A modo de ejemplo, aún existen discriminaciones de género en cuanto a los niveles de ingresos. Esta es la principal conclusión del artículo "La estructura ocupacional de las mujeres en España" escrito por Félix Requena y realizado dentro del grupo de investigación formado por el proyecto SEJ2004-00447 del Ministerio de Educación y Ciencia (Requena, 2008).
} 
también existen barreras internas para ambos géneros. Por ejemplo, la dificultad de las mujeres de negociar la parte material de su retribución y de solicitar aumento de salario. (Kaufmann, 2007).

En definitiva, se da la paradoja de que, mientras por un lado se requiere de competencias emocionales de los profesionales para las que a priori las mujeres pudieran tener una ventaja competitiva, por otro lado también conviven desigualdades de género que se reflejan no únicamente en variables como los ingresos económicos sino también en el acceso a puestos de responsabilidad al interior de las organizaciones.

Al hilo de ello, es interesante resaltar que si bien las mujeres cuentan con un nivel de formación mayor que los hombres (Requena, 2008), no es menos cierto que uno de los requisitos básicos para el acceso a los puestos directivos lo constituye el acceso a programas de master de las escuelas de negocios, y aún la presencia de las potenciales directivas suele ser muy baja, bien por los elevados costes, las incompatibilidades de horarios con la vida personal, como por el estilo agresivo y competitivo de los mismos (Kaufmann, 2007: 127).

También es importante destacar, que si bien otro factor importante para alcanzar los puestos de responsabilidad es la formación en habilidades directivas ${ }^{19}$, las mujeres aún siguen recibiendo poca formación al respecto, aspecto que implica a su vez el desarrollo de la visión estratégica de largo plazo frente a la visión de realizar trabajos (Kaufmann, 2007: 127).

Las desigualdades de género en las organizaciones responden a aspectos complejos y que también implican un cuestionamiento de las relaciones de género fuera del ámbito organizativo. Si bien la igualdad está salvaguardada por las leyes, los hábitos del pasado, los sesgos e incluso las desconfianza hacen que se priorice, consciente o inconscientemente, a los varones sobre las mujeres en los procesos de selección y promoción, anulando y ralentizando los efectos de las leyes igualitarias. Además, la responsabilidad en el cuidado de los hijos y las personas dependientes que aún no es

${ }^{19}$ La formación en habilidades directivas implica los procesos de negociación, de toma de decisiones, de gestión del tiempo, entre otros. 
asumida igualitariamente por los géneros hace que recaiga más en las mujeres y por lo tanto tenga que reducir el tiempo y energía en el desarrollo de su profesión (Díaz, 2008: 9).

Finalmente, frente al requerimiento de un estilo de liderazgo más comunitario, un modelo organizativo más afín al desarrollo de competencias emocionales y de trabajo en equipo, se convive con barreras estructurales tanto en el ámbito doméstico como laboral que posibilitan que las mujeres tengan que realizar doble esfuerzo para lograr la igualdad en el ámbito organizativo.

En síntesis, las cuatro paradojas de la era flexible son resumidas en el siguiente cuadro:

Cuadro 1: Las paradojas del contexto organizativo en la era flexible

\section{Paradojas de la era flexible}

\begin{tabular}{|c|c|}
\hline Oportunidades & Vulnerabilidades \\
\hline $\begin{array}{l}\text { - La reinvención constante del individuo. } \\
\text { - Trayectorias diversas a través de la vida por } \\
\text { proyectos. }\end{array}$ & $\begin{array}{l}\text { - Fragmentación del individuo. } \\
\text { - Emociones vinculadas a la incertidumbre, } \\
\text { soledad, miedo, ansiedad. }\end{array}$ \\
\hline $\begin{array}{l}\text { - Competencias y habilidades de comunicación, } \\
\text { gestión emocional. } \\
\text { - Desarrollo de un liderazgo comunitario y } \\
\text { expresivo. } \\
\text { - Oportunidad de conciliación de la vida } \\
\text { profesional y personal por organización de } \\
\text { tiempos. }\end{array}$ & $\begin{array}{l}\text { - Estrés y déficit de sueño. } \\
\text { - Dificultades en la gestión de los tiempos de } \\
\text { trabajo y de descanso. } \\
\text { - Fenómenos asociados a las emociones en las } \\
\text { organizaciones como mobbing. } \\
\text { - Desigualdades de género. }\end{array}$ \\
\hline
\end{tabular}

Fuente: Elaboración propia.

Es en este transito en el cual emerge el coaching como profesión y espacio de intervención en las organizaciones y los profesionales. Ello merece un apartado específico. 


\section{La emergencia del coaching como profesión: de la vulnerabilidad a la oportunidad}

\subsection{Definición y contexto: áreas de intervención}

El coaching es definido por la Federación Internacional de Coaching ${ }^{20}$ como "una relación profesional continuada que ayuda a obtener resultados extraordinarios en la vida, profesión, empresa o negocios de las personas. Mediante el proceso de coaching, el cliente profundiza en su conocimiento, aumenta su rendimiento y mejora su calidad de vida".

Según el Oxford Dictionary, to coach consiste en tutelar, adiestrar y comunicar hechos (Kaufmann, 2008). En esta acepción se incluyen algunas de las competencias del propio $\operatorname{coach}^{21}$, que es definido como un profesional capaz de observar los fenómenos personales y organizativos, reinterpretarlos de un modo más poderoso e intervenir en ellos de la forma más efectiva posible (Echeverría, 2006).

Si en la actual conceptualización del trabajo no se requiriese del potencial de los profesionales, es decir, de las habilidades y competencias y del desarrollo de un tipo de liderazgo más centrado en la subjetividad, no tendría sentido como tal los servicios profesionales del coaching. Por lo tanto, es el contexto sociológico de reinvención del trabajo, de elasticidad en los límites del ámbito laboral y doméstico, de requerimientos de competencias para adaptarse a la era flexible el que posibilita el área de intervención del coaching. En el contexto de la sociedad del conocimiento surgen también nuevas profesiones adaptadas a las necesidades de un mercado de trabajo ágil, y ello hace

${ }^{20}$ La Federación Internacional de Coaching tiene como misión "la contribución para que la profesión del coaching sea realizada desde la transparencia, ética y con estándares de calidad”. Se cuenta con un código deontológico y se han definido las competencias que requieren los coaches para su práctica profesional. (www.icf-spain.com). Visitado el 10 de Enero de 2009.

${ }^{21}$ El coach domina una serie de competencias que están reconocidas por la Federación Internacional de Coaching y que tiene que ver con la generación de confianza, con el dominio de la comunicación efectiva, con el dominio de las preguntas y la fijación de metas, entre otros (Gorroño, 2008). 
posible que podamos hablar de su emergencia como profesión ${ }^{22}$, emergencia que está por desarrollarse aún, al menos en España, e instalarse o no como tal ${ }^{23}$.

Si bien en España la presencia del coaching es aún incipiente, existen enfoques de intervención distintos. Por un lado, el enfoque anglosajón, característico de EEUU y cuyos referentes comienzan su desarrollo en el ámbito deportivo (Gorroño, 2008), el enfoque ontológico o latinoamericano, impulsado por el trabajo de Rafael Echeverría, y finalmente el enfoque europeo, basado en los trabajos de Jonh Withmore.

Estos enfoques de intervención utilizan diversas técnicas y herramientas para ser aplicadas con el objetivo de desarrollar habilidades y competencias en los profesionales. Además, son compartidos los procesos de diagnosis, por un lado, para la reflexión y la pertinente toma de conciencia y, por otro lado, el proyecto de cambio, que implica la identificación de objetivos y el plan de acción para alcanzarlos.

Ahora bien, hacía falta un contexto sociológico y comprensivo de la realidad social de los profesionales desde el que se asentara una disciplina profesional como el coaching.

Pero, ¿por qué se han abordado las paradojas que transitan entre la oportunidad y la vulnerabilidad para explicar el área de intervención del coaching?

En primer lugar, estas paradojas responden a algunas características de la era flexible, y su lectura en clave de oportunidad y vulnerabilidad contempla la cosmovisión

\footnotetext{
${ }^{22}$ El coaching es definido como un servicio profesional, sin embargo es probable que vaya paulatinamente convirtiéndose en profesión si de manera definitiva es aceptada por el mercado y consolidada con estándares éticos y de calidad. Desde la sociología de las profesiones y desde el enfoque de la escuela definidora, lo que define una profesión son elementos como la transmisión y posesión de conocimientos altamente formalizados, que dicha profesión sea un bien para la comunidad, que disponga de mecanismos de control en el ejercicio profesional, además de que dispongan de una organización colegial profesional. (Rodríguez, 2008).

${ }^{23}$ Algunos datos que ilustran la presencia del coaching en España son los proporcionados en la Encuesta Market Coaching 2008 (Arzuaga, Ortiz de Zarate, Picazo y Zappino, 2008). (Disponible en www.icf-es.com). Según esta encuesta, la primera realizada en España, se utilizó una muestra de 198 empresas, de las cuales contestaron 88. De ellas, el 50\% utilizaron servicios de coaching. El 63\% pertenecían al sector servicios. El 75\% de las que utilizan servicios de coaching, lo hacen desde hace menos de 3 años. El 53\% de las que realizan coaching lo hacen para mejorar el desempeño (79\%), la comunicación (72\%) y el liderazgo (64\%).
} 
de las personas más allá de un ideal del yo independiente y autónomo, cercano a la idea de perfección (Hochschild, 2008: 42). Uno de los peligros de la era flexible como ideología es asumir a los individuos, y también a los profesionales, desde una visión neutra y estática, para así, personalizar los acontecimientos sociales. Es decir, el individualismo puede llevar a adjudicarse personalmente el mérito de los logros y a culpabilizarse personalmente por las pérdidas (Hochschild, 2008: 63).

Si desde la sociología, es aceptado que la realidad se construye socialmente, también los individuos como actores sociales se desarrollan a través de la relación con otros y con ellos mismos en los diversos contextos. (Berger y Luckmann, 1995).

De esta forma, contemplar la vulnerabilidad y oportunidad a nivel macro (contexto sociológico) y también a nivel micro (contexto de la persona) nos permite un acercamiento del área de intervención del coaching de una manera realista y también crítica, teniendo en cuenta el contexto social de intervención y una visión del individuo integrada en su entorno social.

En consecuencia, el área de intervención del coaching en las organizaciones transitaría entre la oportunidad de desarrollar habilidades y competencias de los profesionales y el acompañar la posible vulnerabilidad que la propia oportunidad también puede generar, y esto para, o bien gestionarla cuando ya se da, o bien prevenirla desde la conciencia de que esta vulnerabilidad habita los escenarios organizativos de la eraflexible. 
Esta reflexión se sintetiza en el siguiente cuadro:

Cuadro 2: Áreas de intervención del coaching y metodología de intervención

\section{Áreas de intervención del coaching}

Oportunidades como Competencias

- La reinvención constante del individuo.

- Trayectorias diversas a través de la vida por proyectos.

- Competencias y habilidades de - Prevención y gestión del estrés y déficit comunicación, gestión emocional.

- Desarrollo de un liderazgo comunitario y expresivo.

- Oportunidad de conciliación de la vida profesional y personal por organización de tiempos.
Vulnerabilidades como Prevención y Gestión

- Prevención de la fragmentación

- Gestión de la incertidumbre, soledad, miedo, ansiedad. de descanso.

- Gestión del tiempo de trabajo y de descanso.

-Gestión de la red social en las vulnerabilidades más colectivas: mobbing, desigualdades de género.

\section{Metodología de intervención}

Estado Deseado
Proyecto de cambio

Objetivos

Plan de acción

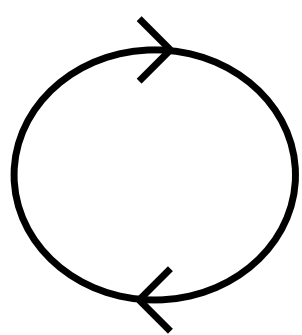

Estado Actual

Diagnosis

Reflexión -Toma de

Conciencia

Fuente: Elaboración propia. 


\section{Algunas consideraciones finales}

De alguna manera, el reflexionar en torno a las características de la era flexible, el cambio de paradigma en el que estamos inmersos y lo que supone ello en el trabajo, devuelve un contexto sociológico a la práctica del coaching. La revisión del contexto en clave de oportunidad y vulnerabilidad abre nuevas posibilidades que nos devuelven a los profesionales como seres humanos imperfectos que sienten el contexto y las consecuencias del mismo. Este sentido de la imperfección toma en consideración que la reinvención constante también implica vulnerabilidad, por lo tanto desde la aceptación de la misma se puede romper el mito de la perfección del yo en ocasiones desarrollado en la literatura de la nueva era y el desarrollo personal. (Hochschild, 2008).

Hemos observado, de manera genérica, algunos entresijos del ámbito del trabajo en transición. También pudiera ser que sus consecuencias se manifestaran en otros ámbitos o incluso en futuras generaciones. Siguiendo el concepto de dolor reflejo (Hochschild, 2008:14), de igual manera que en el cuerpo un síntoma en el hombro puede significar una dolencia más profunda asociada a distintos hábitos sostenidos en el tiempo, entre otras explicaciones, lo sentido en el ámbito del trabajo puede ser síntoma de una dolencia que puede ser reflejada en otro ámbito, más aún cuando vivimos en la vulnerabilidad intangible de las emociones y los sentimientos. Ello también implica desarrollar estrategias de autocuidado importantes, porque ¿dónde están los limites de nuestra propia interioridad?

Además, como apunta Sennet, "un régimen que no proporciona a los seres humanos ninguna razón profunda para cuidarse entre sí no puede preservar por mucho tiempo su legitimidad" (Sennet 2000: 155).

Al hilo de ello, el concebir a las personas desde su imperfección, también devuelve la importancia de las relaciones sociales no únicamente en clave de instrumentalización y rentabilidad en esta era flexible, sino también en clave de conectividad y prevención. Si bien los vínculos sociales pueden ser reconocidos como 
frágiles, retomando a Maffesioli ${ }^{24}$, podemos estar ante la emergencia de la socialidad que integra efectivamente lo sensible, lo imaginario, lo lúdico, lo onírico. Los vínculos frágiles de la era flexible se vuelven emocionales, también líquidos. En vez de temerlos, puede ser una oportunidad desde la cual emerjan nuevas propuestas que obligue a cambiar las lentes con las que se mira la realidad.

Por otro lado, las desigualdades de género en el ámbito organizativo renuevan debates sobre la conciliación de la vida personal y profesional, la reconceptualización del trabajo y la reinvención de nuevas identidades de género más acordes con las necesidades y la complejidad de los tiempos actuales. Ello implica procesos de negociación y cambios profundos que también renuevan las estructuras culturales y climas organizativos.

Finalmente, la emergencia del coaching como profesión dependerá de su consolidación o no en el mercado en función de su utilidad, del rigor profesional y ético y de que se evalúen los resultados obtenidos. Estos han de satisfacer a los profesionales en su propio contexto y también a las organizaciones. Un puzzle aún por resolver.

\footnotetext{
${ }^{24}$ Según el autor, lo social sería lo que reposa únicamente en la razón, el trabajo, el progreso o la fe en el porvenir, lo que constituye las instituciones sociales; frente a la socialidad que estaría caracterizada por vínculos sociales emocionales, espontáneos y esporádicos en el tiempo.
} 


\section{BIBLIOGRAFÍA}

- Arsuaga, E., Ortiz de Zárate, M., Picazo, L., Zappino R. (2008): Market Coaching 08. Disponible en www.icf-es.com

- Bauman, Z. (2007): Vida líquida. Madrid: Paidós Estado y Sociedad ,143.

- Beck, U. (2006): La sociedad del riesgo: hacia una nueva modernidad. Barcelona: Paidós.

- Bell, D. (2001): El advenimiento de la sociedad post-industrial. Madrid: Alianza Universidad.

- Berger, P y Luckmann, T. (1995): La construcción social de la realidad. Buenos Aires: Amorrortu Editores, S.A.

- Boltansky, L y Chiapello E. (2002): El nuevo espíritu del capitalismo. Madrid: Akal

- Castell, M. (2001): La era de la información: economía, sociedad y cultura. La sociedad Red. Madrid: Alianza.

- Cuadrado, I., Navas, M. y Molero, F. (2006), Mujeres y liderazgo: claves psicosociales del techo de cristal. Madrid: Sanz y Torres.

- Díaz, C. (2008): Mujeres en la dirección: la carrera profesional de las mujeres en la empresa, la administración y la universidad. Madrid: Instituto de la mujer. 48.

- Echeverría, R. (2007): La empresa emergente: la confianza y los desafíos de la transformación. Buenos Aires: Granica.

- Echeverría, R. (2006): "Fuenteovejuna o el secreto de los equipos de alto desempeño". Disponible:http://www.portaldelcoaching.com/Actualidad/Columna/docs/Fuente_Oveju na_Rafael_Echeverria.doc

- Fernández, C. (2007): El discurso del Management: tiempo y narración. Madrid: CIS Gasalla, J.M. (2006): La nueva dirección de personas: marco paradójico del talento directivo. Madrid: Pirámide.

- Giddens, A. (2001): Sociología. Madrid: Alianza Editorial.

- Goleman, D. (1998): La práctica de la inteligencia emocional. Barcelona: Cairos. 
- Gorroño, I. (2008): “Coaching y mujeres: nuevos retos para las organizaciones que aprenden”, en P. Rodríguez (Ed.), Mujeres, trabajos y empleos en tiempos de globalización, Barcelona, Icaria, 153-176.

- Gorroño, I. (2008): “El abordaje de las emociones en las organizaciones: luces y sombras", en Domesticación del trabajo, Madrid, Revista de Relaciones laborales, 139. 158.

- Hochschild, A. (2008): La mercantilización de la vida íntima. Buenos Aires: Katz.

- Kaufmann, A. (2007): Mujeres directivas: transición hacia la alta dirección. Madrid: CIS. Opiniones y Actitudes. 56.

- Kaufmann, A y De Prado, M. (2003): Construir equipos de trabajo en la era de la conexión. Madrid: Ensayos y Documentos Editorial.

- Kaufmann, A y Cubeiro J.C (2008): Coaching y diversidad. Madrid: Lid

- Lipovetsky, G. (2007): La felicidad paradójica: ensayo sobre la sociedad del hiperconsumo. Barcelona: Anagrama.

- Martín-Palomo, M. T. (2008): "Domesticación del trabajo: una propuesta para abordar los cuidados", en P. Rodríguez (Ed.), Mujeres, trabajos y empleos en tiempos de globalización, Barcelona, Icaria, 53-86.

- Maffesoli, M. (2004): El tiempo de las tribus. Madrid: Siglo XXI.

- Ministerio de Trabajo y Asuntos Sociales (2006): Encuesta de calidad de vida en trabajo. Disponible en www.mtas.es

- Ministerio de Trabajo y Asuntos Sociales. (2007): Encuesta nacional de condiciones de trabajo. Disponible en www.mtas.es

- Méndez, M.A. (2009): Forzados al reciclaje: El paro y la insatisfacción laboral provocan el cambio de profesión. El País: 8 de Marzo de 2009.

- Requena, F. (2008): “La estructura ocupacional de las mujeres en España”, en P. Rodríguez (Ed.), Mujeres, trabajos y empleos en tiempos de globalización, Barcelona, Icaria, 27-51. 
- Rodríguez, N. (2008): "Policías, arquitectas y abogadas: análisis de tres profesiones", en P. Rodríguez (Ed.), Mujeres, trabajos y empleos en tiempos de globalización, Barcelona, Icaria, 309-328.

- Sarrió, M. (2008): “Coaching y empoderamiento: estrategias para la gestión de la diversidad" en Kaufmann, A y Cubeiro J.C (2008): Coaching y diversidad. Madrid: Lid, 175-201.

- Sennet, R. (2000): La corrosión del carácter. Barcelona: Anagrama.

\title{
Fuentes
}

\author{
Sitios WEB \\ www.icf-spain.com \\ www.acosomoral.org \\ www.mtas.es
}

